\title{
ISHORT COMMUNICATION
}

\section{Impact of thrombus aspiration on the activity of plasminogen activator inhibitor-1 during acute ST-segment elevation myocardial infarction}

\author{
Marin Pavlov'1, Vjeran Nikolić-Heitzler1, Zdravko Babić1,2, Krešimir Kordić1, Ivana Ćelap³, Vesna Degoricija2,4 \\ 1 Department of Cardiology, Sestre Milosrdnice University Hospital Center, Zagreb, Croatia \\ 2 University of Zagreb, School of Medicine, Zagreb, Croatia \\ 3 Department of Clinical Chemistry, Sestre Milosrdnice University Hospital Center, Zagreb, Croatia \\ 4 Department of Medicine, Sestre Milosrdnice University Hospital Center, Zagreb, Croatia
}

Correspondence to: Marin Pavlov, MD, PhD, Department of Cardiology, Sestre Milosrdnice University Hospital Center, Vinogradska cesta 29, 10000 Zagreb, Croatia, phone: +385992360286, email: marin.pavlov@gmail.com Received: September 8, 2020.

Revision accepted: December 11, 2020 Published online: December 11, 2020. Kardiol Pol. 2020; 78 (12): 1281-1283 doi:10.33963/KP.15707 Copyright by the Author(s), 2020
Introduction Restoring a normal coronary flow during acute ST-segment elevation myocardial infarction (STEMI) remains the main objective of interventional cardiology. Historically, thrombus aspiration (TA) was expected to resolve the excessive thrombus dispersion to the coronary microcirculation during the primary percutaneous coronary intervention (PCI). Initial studies suggested that unfavorable blush grades after primary PCI could be avoided by means of TA. ${ }^{1}$ However, subsequent trials showed no consistent benefit of the routine use of TA, even at the expense of excess in stroke. ${ }^{2,3}$ The reason why immediate angiographic benefit does not extend to hard endpoints is not clear.

Plasminogen activator inhibitor-1 (PAI-1) is the central inhibitor of plasminogen activation, deterring endogenous fibrinolysis, and leading to thrombus stabilization or even potential for thrombus extension. Increased PAI-1 activity has been associated with worse short- and long-term outcomes in patients with STEMI. ${ }^{4,5} \mathrm{We}$ hypothesized that TA plays a role in PAI-1 activity dynamics during the acute phase of STEMI.

Methods This was a single-center prospective long-term follow-up study including 87 consecutive patients with acute STEMI treated with primary PCI within 12 hours of the symptom onset (inclusion criterion). ${ }^{5}$ The exclusion criteria were: not available or deceased at 24 hours, chronic corticosteroid or immunosuppressive therapy, no significant coronary artery disease, acute infective or inflammatory disease established within the first 24 hours, active malignant disease and chronic hepatic failure requiring treatment. All patients were participants of the scientific project funded by the Croatian Ministry of Science and Technology (no. 108-1080230-0118). Ethical approval was obtained from both the Central Ethics Committee of the University of Zagreb School of Medicine (04-1387-2006), and the local Sestre Milosrdnice Clinical Hospital Center Ethics Committee (PRO-2-06). All participants signed informed consent prior to enrollment. The enrolment period lasted from May 1, 2009 to March 23, 2010.

Blood samples were collected prior to PCI and after exactly 24 hours. A Berichrom PAI test on the BCS XP analyzer (Siemens, Marburg, Germany) was used for the determination of PAI-1 activity. The PAI- 1 activity rise was calculated as the second sample value subtracted from the pre-PCI value (thus, negative results are allowed). Thrombus aspiration was indicated by an interventional cardiologist. Thrombus burden was determined by the Thrombolysis in Myocardial Infarction (TIMI) thrombus grade ${ }^{6}$ and was documented for the initial diagnostic angiogram (TG1), and, in patients with an occluded coronary artery, after restoring antegrade flow by means of guidewire advancement or small balloon dilatation (TG2). The outcome data after 5 -year follow-up were obtained based on the medical records and a phone call interview with all patients. The composite endpoint was defined as death, stroke, and recurrent acute coronary syndrome. 
TABLE 1 Study population characteristics (unmatched) depending on the use of thrombus aspiration

\begin{tabular}{|c|c|c|c|c|}
\hline \multirow{2}{*}{\multicolumn{2}{|c|}{ Parameter }} & \multicolumn{3}{|c|}{ Thrombus aspiration } \\
\hline & & Yes $(n=12)$ & No $(n=75)$ & $P$ value \\
\hline \multicolumn{2}{|c|}{ Age, y, mean (SD) } & $57.8(11.1)$ & $61.6(12.3)$ & 0.23 \\
\hline \multicolumn{2}{|c|}{ Female sex, $\mathrm{n}(\%)$} & $3(25)$ & $22(29.3)$ & 1 \\
\hline \multirow[t]{5}{*}{ History of } & Hypertension & $6(50)$ & $52(69.3)$ & 0.19 \\
\hline & Diabetes & 0 & $14(18.7)$ & 0.2 \\
\hline & Coronary artery disease & $1(8.3)$ & $10(13.3)$ & 1 \\
\hline & Aspirin use & $3(25)$ & $10(13.5)$ & 0.38 \\
\hline & Statin use & $2(16.7)$ & $4(5.4)$ & 0.19 \\
\hline \multicolumn{2}{|c|}{ Pain-to-first medical contact, min, median (IQR) } & $113(50-166)$ & $123(64-260)$ & 0.22 \\
\hline \multicolumn{2}{|c|}{ First medical contact-to-balloon, min, median (IQR) } & $52(34-66)$ & $60(49-85)$ & 0.11 \\
\hline \multicolumn{2}{|c|}{ Anterior wall myocardial infarction } & $5(41.7)$ & $29(38.7)$ & 1 \\
\hline \multicolumn{2}{|c|}{ Multivessel disease } & $6(50)$ & $45(60)$ & 0.51 \\
\hline \multicolumn{2}{|c|}{ Final TIMI 3 flow } & $10(83.3)$ & $65(86.7)$ & 0.67 \\
\hline \multicolumn{2}{|c|}{ No-reflow phenomenon } & $2(16.7)$ & $21(28)$ & 0.5 \\
\hline \multicolumn{2}{|c|}{ Balloon predilatation } & $2(16.7)$ & $61(81.3)$ & $<0.001$ \\
\hline \multicolumn{2}{|c|}{ Balloon postdilatation } & $1(9.1)$ & $9(12.5)$ & 0.52 \\
\hline \multicolumn{2}{|c|}{ Stent implantation } & $11(91.7)$ & $72(96)$ & 0.51 \\
\hline \multicolumn{2}{|l|}{ Eptifibatide } & $11(91.7)$ & $52(69.3)$ & 0.11 \\
\hline \multicolumn{2}{|c|}{ Unfractionated heparin, U/kg, median, (IQR) } & $77(71-104)$ & $86(71-100)$ & 0.99 \\
\hline \multicolumn{2}{|c|}{ Initial troponin T, ng/l, median (IQR) } & $20(10-51)$ & $87(31-217)$ & 0.02 \\
\hline \multicolumn{2}{|c|}{ Peak creatine kinase, U/I, median (IQR) } & $3146(2158-4067)$ & 2579 (1464-4537) & 0.67 \\
\hline \multicolumn{2}{|c|}{ Occurrence of heart failure } & $1(8.3)$ & $8(10.7)$ & 1 \\
\hline \multicolumn{2}{|c|}{ Cardiogenic shock } & 0 & $3(4)$ & 1 \\
\hline \multicolumn{2}{|c|}{ Ventricular fibrillation } & $2(16.7)$ & $9(34.2)$ & 0.64 \\
\hline \multicolumn{2}{|c|}{ Creatinine clearance, ml/kg, mean (SD) } & $60(22)$ & $58(21)$ & 0.52 \\
\hline \multicolumn{2}{|c|}{ Left ventricular ejection fraction, $\%$, median (IQR) } & $50(48-55)$ & $55(45-57)$ & 0.62 \\
\hline \multicolumn{2}{|c|}{ Body mass index, kg/m², mean (SD) } & $27.2(5.5)$ & $27.8(3.7)$ & 0.69 \\
\hline
\end{tabular}

Data are presented as number (percentage) of patients, unless indicated otherwise.

Abbreviations: IQR, interquartile range; TIMI, thrombolysis in myocardial infarction

Statistical analysis Statistical analysis was performed using the SPSS for Windows (IBM SPSS, version 25, Armonk, New York, United States). Continuous variables were tested for normality of distribution by the Kolmogorov-Smirnov test. They were expressed as means (SD) or medians and interquartile ranges, and analyzed by the Mann-Whitney test. Categorical variables were expressed as counts and frequencies, and were assessed by $\mathrm{X}^{2}$ or the Fisher exact test. Correlations were tested by the Spearman correlation coefficient. The binary logistic regression was used to determine independent contribution of individual variables to increased PAI-1 activity (an optimal cutoff point for dichotomization was determined by the receiver-operating characteristic curve analysis). ${ }^{5}$ The results are presented as odds ratio (OR) with 95\% CIs. Continuous PAI-1 activity levels were additionally analyzed after propensity score matching (one-to-one matching for age, sex, diabetes, heart failure, shock, and anterior wall infarction; without replacement; tolerance of 0.005). For matching and regression analysis, variables related with an increase in PAI-1 activity in univariate analysis or clinically relevant variables were used. A 2-tailed significance tests were performed, $P$ values lower than 0.05 were considered significant.

Results and discussion Study population characteristics are presented in TABLE 1. Balloon angioplasty was not associated with increased PAI-1 activity. No correlation was found between balloon-to-stent ratio and an increase in PAI-1 activity 
( $\rho=0.173, P=0.19)$. In patients treated with TA, a greater increase in PAI-1 activity was detected (median [IQR], 3.25 [1.6-4.55] U/ml vs 1.2 [-0.2 to 2.2$] \mathrm{U} / \mathrm{ml} ; P=0.003)$. The increase in PAI-1 activity differed among groups of patients with different interventional procedures employed, with the highest value in the group treated with both balloon angioplasty and TA $(P=0.02)$.

TIMI thrombus grades (TG1 and TG2) were not associated with the increase in PAI- 1 activity $(P=0.29$ and $P=0.6)$. Both TG1 and TG2 values showed no difference regarding the use of TA $(P=0.08$ and $P=0.97)$.

In a stepwise binary logistic regression model with 9 variables (age, sex, total ischemic time, TA, heart failure, no-reflow, final TIMI flow, peak creatine kinase, and body mass index), independent predictors of increased PAI-1 activity higher than $3.7 \mathrm{U} / \mathrm{ml}$ were female sex (OR, 11.9; 95\% CI, 2.1-66.1; $P=0.005)$, TA (OR, 13.1; 95\% CI, 2.1-83.3; $P=0.006$ ), and heart failure (OR, 29.3; 95\% CI, 3.8-225.2; $P=0.001)$.

Two patients (2.3\%) died during hospital treatment. Eleven additional deaths (12.6\%) and 18 composite endpoint events (20.7\%) occurred during follow-up. No difference in endpoint occurrence was found between the groups treated and not treated with TA $(P=0.38$ and $P=0.71)$.

After propensity score matching, 24 cases remained in the analysis. The increase in PAI-1 activity was higher in patients treated with TA (median [IQR], 3.24 [1.29-4.72] U/ml vs 1.45 [0.42-2.46] U/ml; $P=0.03)$.

A greater increase in PAI-1 activity was detected in patients in whom TA was used. The difference persisted after propensity score matching. In the binary logistic regression, TA was independently related to the increase in PAI- 1 activity. Thrombus disintegration is possibly the cause of increased PAI-1 activity, since coronary thrombi are known to contain over 2000 -fold the normal plasma PAI-1 concentration. ${ }^{7}$ However, other means of thrombus disintegration, such as balloon angioplasty, proved not to be related to increased PAI-1 activity. This could signify that TA results in more abundant thrombus disintegration than balloon angioplasty. To the best of our knowledge, this was the first study to assess PAI-1 dynamics in relation to TA and balloon angioplasty. The more aggressive strategy was used, the higher the observed increase in PAI-1 activity. Yet, the use of TA could simply be an indicator of higher thrombotic burden. Hence, the basis for the detected increased PAI- 1 activity could be the extent of the intracoronary thrombotic material. In an attempt to resolve this issue, we used TIMI thrombus grade, which showed no association with increased PAI-1 activity. Contrary to the expectation, TA was not associated with initial TIMI thrombus grades. Therefore, we cannot conclude that thrombotic burden was the origin of increased PAI-1 activity.
Two large randomized trials showed no benefit of routine TA in patients with STEMI. 2,3 Current guidelines still recommend that the method may be considered in selected patients. ${ }^{8}$ Even with the same angiographic finding, the benefit of TA may vary, and is possibly related to total ischemic time. The effect of TA on outcomes resembles a U-shaped curve with the highest benefit for patients with total ischemic time from 4 to 6 hours, ${ }^{9}$ and no benefit in patients presenting late (>12 hours) after symptom onset. ${ }^{10}$ The possible explanation of the increasing benefit of TA over time is that the contribution of distal embolization to microvascular obstruction becomes more relevant in later stages when the thrombus is increasingly organized and more apt to disrupt in insoluble fragments. ${ }^{9}$ Later on, thrombus will become more challenging to retrieve with TA. Interestingly, we found the lowest increase in PAI-1 activity in similar time frame from symptom onset (3-6 hours).

To conclude, TA was associated with a greater increase in PAI-1 activity in patients with acute STEMI treated with primary PCI. The clinical implications of this association should be tested in larger studies.

\section{ARTICLE INFORMATION}

\section{CONFLICT OF INTEREST None declared.}

OPEN ACCESS This is an Open Access article distributed under the terms of the Creative Commons Attribution-NonCommercial-NoDerivatives $4.0 \mathrm{In}$ ternational License (CC BY-NC-ND 4.0), allowing third parties to download articles and share them with others, provided the original work is properly cited, not changed in any way, distributed under the same license, and used for noncommercial purposes only. For commercial use, please contact the journal office at kardiologiapolska@ptkardio.pl.

HOW TO CITE Pavlov M, Nikolić-Heitzler V, Babić Z, et al. Impact of thrombus aspiration on the activity of plasminogen activator inhibitor-1 during acute ST-segment elevation myocardial infarction. Kardiol Pol. 2020; 78: 1281-1283. doi:10.33963/KP.15707

\section{REFERENCES}

1 Svilaas T, Vlaar PJ, van der Horst IC, et al. Thrombus aspiration during primary percutaneous coronary intervention. $N$ Engl J Med. 2008; 358: 557-567.

2 Frobert 0 , Lagerqvist B, Olivecrona GK, et al. Thrombus aspiration during ST-segment elevation myocardial infarction. N Engl J Med. 2013; 369: 1587-1597.

3 Jolly SS, Cairns JA, Yusuf S, et al. Randomized trial of primary PCI with or without routine manual thrombectomy. N Engl J Med. 2015; 372: 1389-1398.

4 Collet JP, Montalescot $\mathrm{G}$, Vicaut E, et al. Acute release of plasminogen activator inhibitor-1 in ST-segment elevation myocardial infarction predicts mortality. Circulation. 2003; 108: 391-394.

5 Pavlov M, Nikolic-Heitzler V, Babic Z, et al. Plasminogen activator inhibitor-1 activity and long-term outcome in patients with ST-elevation myocardial infarction treated with primary percutaneous coronary intervention: a prospective cohort study. Croat Med J. 2018; 59: 108-117.

6 Sianos G, Papafaklis MI, Serruys PW. Angiographic thrombus burden classification in patients with ST-segment elevation myocardial infarction treated with percutaneous coronary intervention. J Invasive Cardiol. 2010; 22: 6b-14b.

7 Fay WP, Murphy JG, Owen WG. High concentrations of active plasminogen activator inhibitor-1 in porcine coronary artery thrombi. Arterioscler Thromb Vasc Biol. 1996; 16: 1277-1284.

8 Ibanez B, James S, Agewall S, et al. 2017 ESC Guidelines for the management of acute myocardial infarction in patients presenting with ST-segment elevation: the Task Force for the management of acute myocardial infarction in patients presenting with ST-segment elevation of the European Society of Cardiology (ESC). Eur Heart J. 2018; 39: 119-177.

9 Sim DS, Jeong MH, Ahn Y, et al. Manual thrombus aspiration during primary percutaneous coronary intervention: impact of total ischemic time. J Cardiol. 2017; 69: 428-435.

10 Freund A, Schock S, Stiermaier T, et al. Thrombus aspiration in patients with ST-elevation myocardial infarction presenting late after symptom onset: long-term clinical outcome of a randomized trial. Clin Res Cardiol. 2019; 108: 1208-1214. 